\title{
ESPONDILOLISTESIS ÍSTMICA LUMBOSACRA DEL ADULTO: LAMINOARTRECTOMÍA Y FUSIÓN IN SITU
}

\author{
ESPONDILOLISTESE ÍSTMICA LOMBOSSACRAL DO ADULTO: LÂMINO-ARTRECTOMIA \\ E FUSÃO IN SITU
}

\section{ADULT LUMBOSACRAL ISTHMIC SPONDYLOLISTHESIS: LAMINECTOMYIARTHRECTOMY AND IN SITU FUSION}

Patricio Manzone ${ }^{1}$, Claudia Ihlenfeld², Eduardo Mariño Ávalos³3, José Felipe Gemetro ${ }^{4}$

\begin{abstract}
RESUMEN
Objetivo: La espondilolistesis ístmica del adulto se puede tratar con reparación del defecto, descompresión (sola o con fusión), fusión circunferencial, y reducción con fijación posterior. El objetivo de este trabajo es valorar los resultados a medio plazo de la descompresión y fusión in situ en pacientes operados por un solo cirujano. Método: Criterios de Inclusión: 1) Espondilolistesis Istmica L5-S1. 2) Operados en madurez esquelética. 3) Laminoartrectomía de L5 con Artrodesis posterolateral instrumentada sin reducción. 4) Más de 3 años de seguimiento. 5) Ausencia de pseudoartrosis al seguimiento. 6) Valoración funcional y radiológica por observador independiente. Comparación de datos con bibliografía. Análisis estadístico con t - test, Chi cuadrado y ANOVA. Resultados: 16 pacientes (12 \&/4 $\left.\jmath^{1}\right)$ evaluados. Edad promedio: 40,6 años (17 - 66); seguimiento promedio: 10.3 años (3.3 - 18). Deslizamiento promedio: 42,9\%. Nueve presentaban dolor radicular, 3 dolor lumbar y 4 dolor lumborradicular. Score del dolor (SRS) preoperatorio promedio: 1.38 , sin diferencias entre bajo y alto grado ( $p=0.887$ ). $15 / 16$ mejoraron (score final promedio: 4.44$)$. 13/16 retornaron a su actividad previa (81\%). Hubo 2 infecciones; no hubo complicaciones neurológicas. Los parámetros espinopélvicos finales, comparados con la literatura no mostraron diferencias estadísticas. La comparación entre listesis de bajo y alto grado en la serie no mostró diferencia significativa en dichos parámetros, ni en tiempo de seguimiento, niveles artrodesados, síntomas ni retorno al trabajo. Conclusión: La descompresión y fusión posterolateral instrumentada in situ permite un $80 \%$ de buenos resultados en la espondilolistesis ístmica lumbosacra del adulto sin importar el grado.
\end{abstract}

Descriptores: Espondilolistesis, Cirugía; Artrodesis; Laminectomía; Fusión vertebral.

\begin{abstract}
RESUMO
Objetivo: A espondilolistese ístmica dos adultos pode ser tratada com reparo do defeito, descompressão (isolada ou com fusão), fusão circunferencial e redução com fixação posterior. O objetivo foi avaliar os resultados a médio prazo da descompressão e fusão in situ em pacientes submetidos a tratamento cirúrgico por um só cirurgião. Método: Critérios de inclusão: 1) Espondilolistese ístmica L5-S1. 2) Cirurgia depois da maturidade esquelética. 3) Lâmino-artrectomia e artrodese póstero-lateral instrumentada em L5 sem redução. 4) Mais de três anos de acompanhamento. 5) Ausência de pseudoartrose no acompanhamento. 6) Avaliação funcional e radiológica por observadores independentes. Comparação com a literatura. Análise estatística por teste "t", qui quadrado e ANOVA. Resultados: Foram avaliados 16 pacientes (12 9 e 4 ô). Média de idade: 40,6 anos (17-66), acompanhamento médio: 10,3 anos (3,3-18). Média do deslizamento: 42,9\%. Nove tinham dor radicular, 3 lombar, 4 dor radicular e lombar. Pontuação de dor pré-operatória média (segundo SRS): 1,38, sem diferenças entre baixo e alto grau $(p=0,887)$. Quinze em 16 pacientes tiveram melhora dos sintomas (pontuação final média: 4,44). Treze em 16 pacientes retornaram à atividade anterior (81\%). Houve duas infecções, mas nenhuma complicação neurológica. Os parâmetros espino-pélvicos no final do acompanhamento não apresentaram diferenças estatísticas em relação com os valores encontrados na literatura. A comparação entre deslizamentos de baixo e alto grau da série não mostrou diferença significante nesses parâmetros nem em tempo de acompanhamento, níveis com artrodese, sintomas ou retorno ao trabalho. Conclusão: A descompressão póstero-lateral e fusão instrumentada in situ permitem $80 \%$ de bons resultados na espondilolistese ístmica lombossacral em adultos, independentemente do grau de deslizamento.
\end{abstract}

Descritores: Espondilolistese, Cirurgia; Artrodese; Laminectomia; Fusão Vertebral.

\begin{abstract}
Objective: Adult isthmic spondylolisthesis can be treated with direct repair of the defect, decompression (alone or with posterolateral fusion), circumferential fusion, and reduction with posterior fixation. The aim of this work is to assess the mid-term results of decompression and in situ fusion among patients undergoing surgical treatment with the same surgeon. Method: Inclusion criteria: 1) L5-S1 Isthmic spondylolisthesis. 2) Surgery performed after skeletal maturity. 3) L5 laminectomy and arthrectomy with posterolateral instrumented fusion without reduction. 4) Follow-up greater than 3 years. 5) No pseudoarthrosis at follow-up. 6) Functional and radiological assessment by independent observers; literature review and data comparison; statistical analysis with t-test, chi-squared and ANOVA. Results: 16 patients were evaluated (12 +/4 ô). Mean age: 40.6 years (17-66); mean follow-up: 10.3 years (3.3-18). Average slip: 42.9\%. Nine patients had radicular pain, 3 back pain and 4 both. Mean preoperative SRS pain score: 1.38 with no differences between low and high degree $(p=0.887)$. Fifteen of 16 patients had improvement of symptoms (mean final score: 4.44$)$. Thirteen of 16 patients returned to previous activity (81\%). There were 2 infections, but no neurologic complications. The spino-pelvic parameters at the end of the follow-up showed no statistical differences compared with the values found in literature. The comparison between low and high degree listhesis in our series showed no significant difference in these parameters or in the follow-up period, fused levels, symptoms or return to work. Conclusion: The posterolateral decompression and instrumented fusion in situ allow $80 \%$ of good results in lumbosacral isthmic spondylolisthesis in adults regardless of the degree of slip.
\end{abstract}

Keywords: Spondylolisthesis, Surgery; Arthrodesis; Laminectomy; Spinal fusion.

1. Diretor Medico - Centro "Nicolas Andry" - Monteagudo 207 - (3500) Resistencia - Argentina.

2. Médico - Centro "Nicolas Andry" - Monteagudo 207 - (3500) Resistencia - Argentina.

3. Médico - Instituto Médico "Dra. Kyra Popowitch"

4. Médico Felipe Gemetro - Hospital "4 de Junio Dr. Ramón Carrillo"

Trabajo realizado no Centro "Nicolas Andry" - Monteagudo 207 - (3500) Resistencia - Argentina.

Correspondência: Institución: Centro "Nicolas Andry" - Monteagudo 207 - (3500) Resistencia - Argentina. E-mail: manzonepatricio@ hotmail.com

Recebido em 31/01/2012, aceito em 04/07/2012. 


\section{INTRODUCCIÓN}

La espóndilolistesis lumbosacra ístmica del adulto se puede tratar con reparación del defecto, descompresión (sola o con fusión póstero-lateral), fusión intersomática anterior aislada, fusión circunferencial y reducción con fijación posterior ${ }^{1,2}$.

En pacientes apropiadamente seleccionados se puede tener un $80 \%$ o más de resultados satisfactorios independientemente de la técnica usada².

El objetivo del presente trabajo fue valorar los resultados a mediano plazo de la descompresión y fusión in situ en pacientes operados por un solo cirujano en un mismo centro.

\section{MATERIAL Y MÉTODOS}

Los Criterios de Inclusión para este trabajo fueron: 1) Pacientes con Espondilolistesis Ístmica L5-S1, tipo lítica a partir de Risser 5, 2) operados después de la madurez esquelética, 3) con Laminoartrectomía de L5 y Artrodesis posterolateral in situ instrumentada, es decir sin reducción o maniobras de corrección instrumental, 4) con más de 3 años de seguimiento, 5) ausencia de Pseudoartrosis al seguimiento, 6) Valoración funcional y radiológica por observador independiente, de 7) Espinogramas y Rx focalizadas de columna lumbosacra y pelvis al final del seguimiento.

Se efectuó un Análisis Estadístico usando como pruebas no paramétricas el t-test de Student y la ANOVA para las variables cuantitativas, y el Chi cuadrado y el test de Fischer para las cualitativas. Se aceptó como valor significativo de p a partir de 0.05.

En las radiografías preoperatorias y en los espinogramas y radiografías del seguimiento fueron medidas las desalineaciones en el plano frontal de columna dorsal y lumbar, la cifosis dorsal, la lordosis lumbar, el ángulo lumbosacro, el grado de listesis, el porcentaje de deslizamiento, el porcentaje de corrección, la curva sacra, el índice lumbar, la incidencia pelviana, la pendiente sacra, la inclinación pelviana y el "overhang de S1"; todo ello según los criterios de las publicaciones existentes en la literatura ${ }^{3-21}$.

Se compararon los valores preoperatorios y los valores postoperatorios del seguimiento de nuestra serie entre sí, y también estos con los valores de series de pacientes normales de la bibliografía ${ }^{7,9-13,15,18,21}$

Se compararon también nuestros valores preoperatorios con los valores de series de pacientes con espondilolistesis lumbosacra NO operados de la bibliografía $6-8,11,12,15,16,22$, tanto globalmente como subdivididos en subgrupo de Alto y Bajo Grado de deslizamiento.

Por último, también se compararon los valores postoperatorios del seguimiento de nuestra serie con los valores postoperatorios de series operadas publicadas ${ }^{23-26}$.

\section{RESULTADOS}

Se evaluaron 16 pacientes (12 mujeres/4 varones), con una edad promedio de 40.6 años (rango 16.64 - 66), con un seguimiento promedio de 10.3 años (3.3 - 18 años). El promedio de deslizamiento de la serie fue de $42.9 \%$ (10\% - 100\%), y el grado promedio fue 2.3. La actividad laboral preoperatoria era de escritorio en 12 pacientes, de esfuerzo en 2 y retirados en 2. Nueve (9) pacientes presentaban dolor radicular aislado en preoperatorio,

3 dolor lumbar aislado y 4 dolor lumboradicular. El score del dolor de la SRS en preoperatorio dio un promedio de $1.38(1-2)$, sin diferencias significativas entre pacientes con listesis de bajo grado y alto grado ( $p$ 0.887).

En la cirugía se artrodesaron tres (3) espacios discales [L3-S1] en 1 paciente, dos (2) espacios [L4-S1] en 13 pacientes, y un (1) espacio [L5-S1] en 2 pacientes.

Al seguimiento todos excepto uno mejoraron de su sintomatología: 12 se encontraban asintomáticos, 3 presentaban síntomas episódicos leves pero uno (1) tenía síntomas continuos leves. El score del dolor de la SRS al seguimiento dio en promedio 4.44 (2 - 5), sin diferencias estadísticas significativa entre pacientes con listesis de bajo y de alto grado ( $p$ 0.118). Al seguimiento: 13 pacientes retornaron completamente a su actividad laboral previa (81\%).
Como complicaciones hubo 2 infecciones que curaron con toilettes y antibioticoterapia general (Figura 1a,b,c,d), y una progresión de la listesis con artrodesis posterolateral constituida que obligó a una artrodesis anterior intersomática (Figura 2a,b,c,d,e). No hubo ninguna complicación neurológica. La tasa de complicaciones fue entonces de $18.8 \%(3 / 16)$.

Los valores preoperatorios de los parámetros espino-pélvicos de nuestra serie comparados con los de series de pacientes normales de la literatura mostraron diferencias significativas en todos excepto para la curva sacra (Tabla 1).

Cuando se compararon los valores hallados en preoperatorio en nuestro subgrupo de Espondilolistesis de Alto Grado (> 50\%) con los de series similares de la literatura, la única diferencia significativa que se encontró fue en la inclinación pelviana ( $p$ 0.0043)

En el caso de la comparación de los valores preoperatorios de las Espondilolistesis de Bajo Grado (<50\%) de nuestra serie con los de la bibliografía, o hubo diferencias significativas para la lordosis lumbar $(p<0.0001)$, la curva sacra $(p<0.0001)$ y la pendiente sacra $(p 0.0259)$.
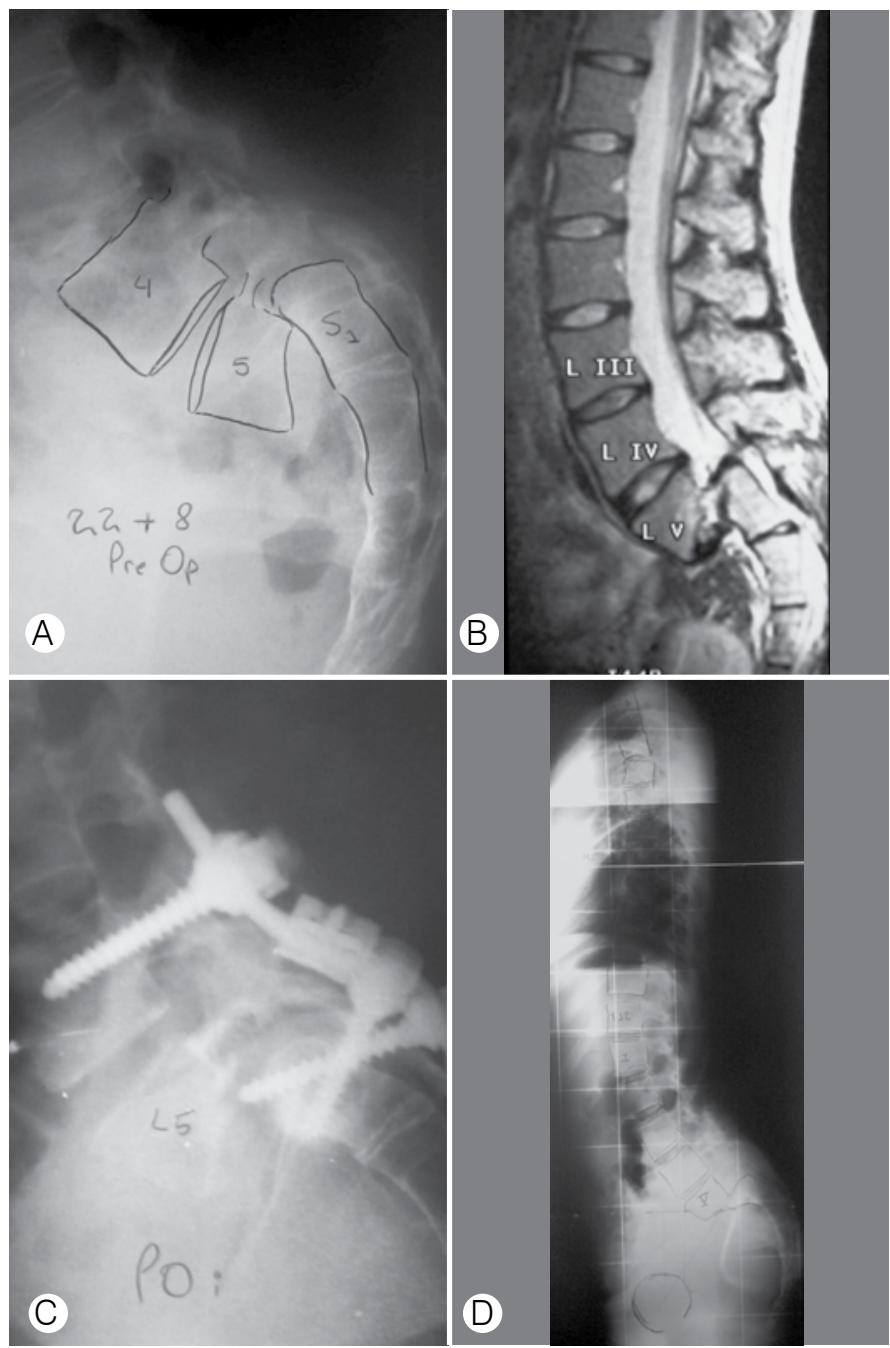

Figura 1. Paciente de 22 años 8 meses, sexo femenino, con espóndiloptosis (Espondilolistesis grado V) con dolor lumbar severo (SRS 2); operada con fusión in situ L4-S1 instrumentada y laminoartrectomía. Complicación: infección del sitio de toma de injerto en cresta ilíaca; cura sin inconvenientes. Requiere extracción de implantes a 5 años de la cirugía original por prominencia de los tornillos sacros. Seguimiento de 11 años 6 meses; sin dolor (SRS 5), ocupación: maestra rural. (A) Rx perfil Preoperatoria; (B) Resonancia Magnética Preoperatoria (corte sagital); (C) Rx perfil Postoperatorio inmediato: nótese la corrección únicamente postural (en mesa de operaciones) de la cifosis lumbosacra y del deslizamiento; (D) Espinograma perfil al seguimiento; nótese la existencia de una fusión anterior espontánea. 


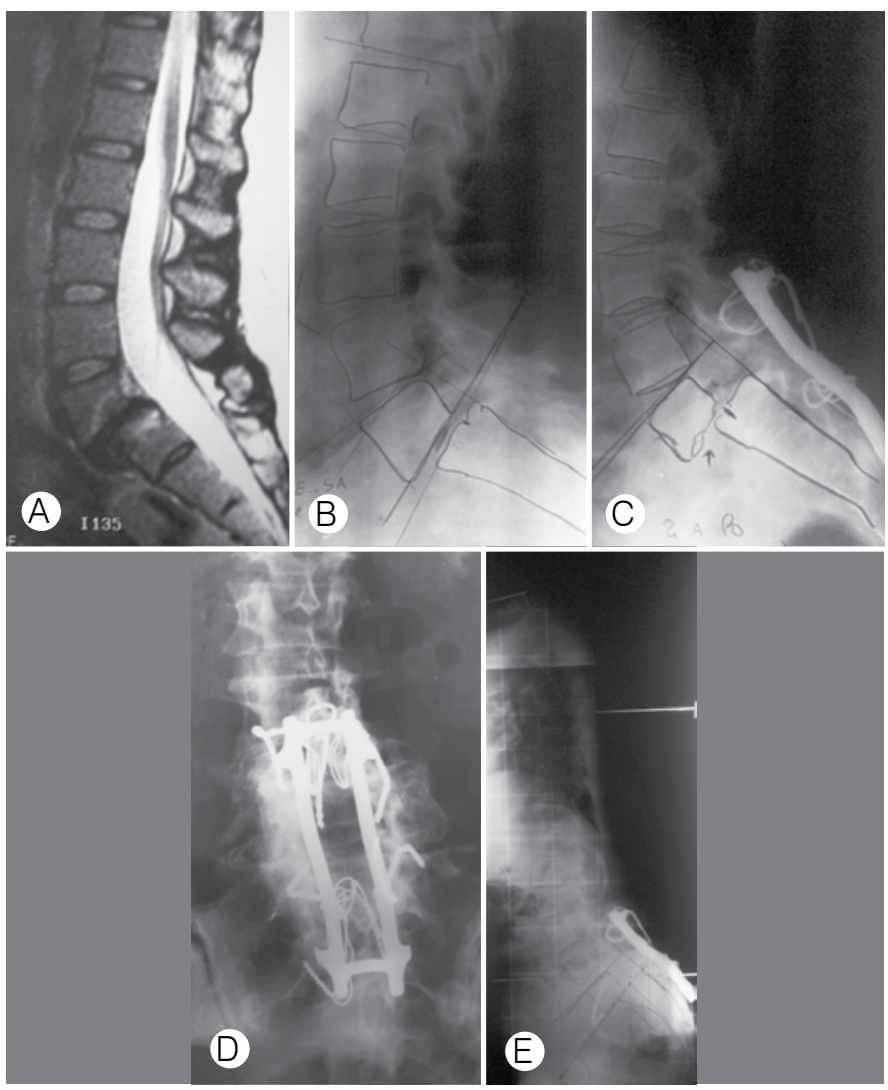

Figura 2. Paciente de 40 años, sexo femenino, con espondilolistesis de grado II con dolor radicular severo en pre-operatorio (SRS 1); operada con fusión in situ L4-S1 instrumentada con Marco con alambres sublaminares, y laminoartrectomía. A pesar de una sólida fusión posterolateral hay progresión sintomática de deslizamiento en postoperatorio pasando a grado III. Se complementa con una fusión intersomática L5-S1 por vía anterior transperitoneal. En postoperatorio alejado (15 años 7 meses PO I; 13 años $6 \mathrm{~m}$ del PO II) presenta una reducción significativa de su dolor (SRS 4), con vuelta parcial a su actividad previa (ama de casa). (A) Resonancia Magnética Preoperatoria; (B) Rx perfil Preoperatoria; (C) Rx Perfil a 2 años de postoperatorio: progresión del deslizamiento; (D) Rx Póstero-anterior a 2 años de postoperatorio: obsérvese la masa de fusión; (E) Espinograma perfil al seguimiento (15 años $7 \mathrm{~m} \mathrm{PO} \mathrm{I;} 13$ años $6 \mathrm{~m}$ del PO II).

Tabla 1. Comparación de los diferentes parámetros preoperatorios y postoperatorios de los pacientes de la presente serie con los valores de los parámetros de series de personas normales publicadas en la literatura $^{3,5,7,9,12,14-16}$

\begin{tabular}{c|c|c}
\hline & Valor de p en pre-operatorio & Valor de p en post-operatorio \\
\hline Iordosis Lumbar & $<0.0001\left(^{*}\right)$ & $<0.0001\left(^{*}\right)$ \\
\hline Curva Sacra & 0.068 & 0.29 \\
\hline Incidencia Pélvica & $<0.0001\left(^{*}\right)$ & $<0.0001\left(^{*}\right)$ \\
\hline Pendiente sacra & $<0.0001\left(^{*}\right)$ & $<0.0001\left(^{*}\right)$ \\
\hline Inclinación Pélvica & $<0.0001\left(^{*}\right)$ & $<0.0001\left(^{*}\right)$ \\
\hline Ángulo Lumbosacro & $0.015\left(^{*}\right)$ & $0.0076\left({ }^{*}\right)$ \\
\hline Edad & $0.001\left(^{*}\right)$ & $S D$ \\
\hline$\left.{ }^{*}\right)$ : Estadísticamente significativo. SD: sin datos.
\end{tabular}

En la comparación de las mediciones al seguimiento de nuestra serie con los controles normales de la literatura obviamente siempre hubo diferencias estadísticamente significativas puesto que no se realizó corrección (Tabla 1). En el único valor donde no hubo diferencias fue en la curva sacra.

Nuestros parámetros espino-pélvicos finales comparados con las de series de espondilolistesis operadas en la literatura, no mostraron diferencias significativas en ninguno de ellos salvo en el porcentaje de deslizamiento y la edad - ambas mayores en nuestra serie (Tabla 2).

Por último, al comparar dentro de nuestra propia serie los pacientes con listesis de bajo grado y de alto grado, en preoperatorio sí encontramos obviamente diferencias estadísticamente significativas en el porcentaje y grado de listesis $v$-como era de esperar- aunque también en la inclinación pélvica; pero no hubo diferencias significativas en los restantes parámetros ni en la edad a la cirugía, sexo y actividad laboral preoperatoria (Tabla 3). En postoperatorio, la comparación estadística entre ambos subgrupos no mostró ninguna diferencia significativa en ninguno de los parámetros espino-pélvicos salvo nuevamente en porcentaje y grado de deslizamiento; tampoco hubo diferencias en tiempo de seguimiento, niveles artrodesados, síntomas postoperatorios y retorno postoperatorio a la actividad laboral (Tabla 3).

Tabla 2. Comparación entre los parámetros postoperatorios de la presente serie y de serie operada de la literatura ${ }^{23}$

\begin{tabular}{c|c}
\hline & Valor de $\mathbf{p}$ \\
\hline Lordosis lumbar & 0.22 \\
\hline Incidencia Pélvica & 0.14 \\
\hline Pendiente Sacra & 0.89 \\
\hline Inclinación Pélvica & 0.21 \\
\hline Ángulo Lumbosacro & 0.82 \\
\hline Porcentaje de Deslizamiento & $0.002\left({ }^{*}\right)$ \\
\hline Edad a la cirugía & $0.0001\left(^{*}\right)$
\end{tabular}

Tabla 3. Comparación de los diferentes parámetros entre los pacientes de Bajo (menor o igual a $50 \%$ ) y Alto Grado (> de $50 \%$ ) de deslizamiento de la presente serie. Valor de $\mathrm{p}$.

\begin{tabular}{c|c|c}
\hline & $\begin{array}{c}\text { Valor de p en } \\
\text { Preoperatorio }\end{array}$ & $\begin{array}{c}\text { Valor de p en } \\
\text { Postoperatorio }\end{array}$ \\
\hline Grado de Listesis & $<0.0001\left(^{*}\right)$ & $0.014\left(^{*}\right)$ \\
\hline Porcentaje de Listesis & $0.0003\left(^{*}\right)$ & $0.028\left(^{*}\right)$ \\
\hline Lordosis Lumbar & 0.26 & 0.22 \\
\hline Cifosis Lumbosacra & 0.6 & 0.97 \\
\hline Curva Sacra & 0.17 & 0.69 \\
\hline Indice Lumbar & 0.37 & 0.58 \\
\hline Incidencia Pelvica & 0.44 & 0.71 \\
\hline Pendiente sacra & 0.63 & 0.6 \\
\hline Inclinacion Pelvica & $0.01\left(^{*}\right)$ & 0.075 \\
\hline Edad a la cirugia & 0.07 & $\mathrm{NA}$ \\
\hline Sexo & 1 & 0.31 \\
\hline Tiempo de Seguimiento & $\mathrm{NA}$ & 0.24 \\
\hline Niveles Artrodesados & $\mathrm{NA}$ & 0.09 \\
\hline Presencia de Sintomas Preoperatorios & $\mathrm{NA}$ & 0.11 \\
\hline Retorno a la Actividad Laboral & $\mathrm{NA}$ &
\end{tabular}

(*): Estadísticamente significativo. NA: No aplicable.

\section{DISCUSIÓN}

Además de la fusión postero-lateral in situ, con o sin descompresión asociada, diversas técnicas son actualmente preconizadas para el tratamiento de la espondilolistesis lumbosacra ístmica sintomática del adulto ${ }^{1,2,27-33}$. Todas con buenos resultados en los diferentes reportes.

Sin embargo, los riesgos de morbi-mortalidad en el tratamiento quirúrgico de estos casos, si bien bajos no son desdeñables y aumentan a medida que el grado de deslizamiento lo hace ${ }^{34,35}$

Históricamente, la fusión in situ incluso sin instrumentación ha demostrado proveer alrededor de un $80 \%$ de resultados clínicos satisfactorios aún en presencia de pseudoartrosis, siendo discutida por algunos la utilidad del agregado de la descompresión, pero aceptado de manera generalizada la utilidad de la instrumentación con tornillos pediculares ${ }^{36-43}$. Los resultados clínicos de nuestra serie se comparan favorablemente con los de la literatura.

En un artículo de revisión reciente Agabegi y Fischgrund ${ }^{1}$ confirman que la obtención de una sólida fusión lleva a mejores resultados funcionales y de reducción del dolor. También hacen notar que la artrodesis circunferencial se asocia con un mayor índice de fusión y se volvió más común, especialmente en los deslizamientos de alto grado. En nuestra serie, una sola paciente necesitó una fusión anterior para detener un deslizamiento sintomático que proseguía a pesar de una masa de artrodesis posterolateral bien constituida y visible (Figura 2a,b,c,d,e). Dicha evolución se atribuyó a que la instrumentación usada no era con tornillos pediculares sino simplemente de alambrado sublaminar, como ya fue descripto previamente ${ }^{44}$ 
Además, las comparaciones en la literatura de la fusión in situ con técnicas de suplementación anterior desde atrás, especialmente PLIF, no son categóricas en cuanto a la mejoría de los resultados a mediano o largo plazo ${ }^{45-48}$. Lo mismo puede decirse de las artrodesis anteriores puras - ALIF $^{49}$.

Benli et al. ${ }^{48}$ en un estudio prospectivo randomizado de pacientes con fusión posterolateral instrumentada divididos en aquellos con reducción y otros solo con modelado sagital mostraron que una artrodesis exitosa y la descompresión neural son los parámetros más importantes que tienen impacto en el resultado clínico independientemente del grado de listesis y que la reducción no tenía traducción estadística en la mejoría del resultado clínico. Los resultados de nuestra serie coinciden con el hallazgo de estos últimos autores (Figura 3a,b,c).

Si bien existen trabajos sobre las modificaciones en la alineación y el equilibrio espino-pélvico con las técnicas de corrección y reducción de la listesis, sus resultados son aún controvertidos $23,25,26,48,50$. La incidencia pelviana no se modifica con la reducción puesto que es un parámetro individual fijo $11,20,26,51,52$. La necesidad de reducción continúa siendo discutida y de manera mayoritaria parece ser indicada en pacientes con excesiva cifosis lumbosacra y gran desequilibrio espino-pévico 1,16,28. Por otra parte, aparentemente solo la recuperación de la altura discal se correlaciona de manera significativa con un cambio en la lordosis lumbar y en el equilibrio sagital ${ }^{49}$.

Quizás el modelado sagital lumbosacro como propone Benli et al. ${ }^{48}$ y la obtención de una sólida artrodesis como también encuentra este autor, así como lo hace Hresko et al..$^{50}$ para la misma condición pero en adolescentes, sean más importantes que la reducción de la listesis en determinar el balance espino-pélvico sagital postoperatorio.
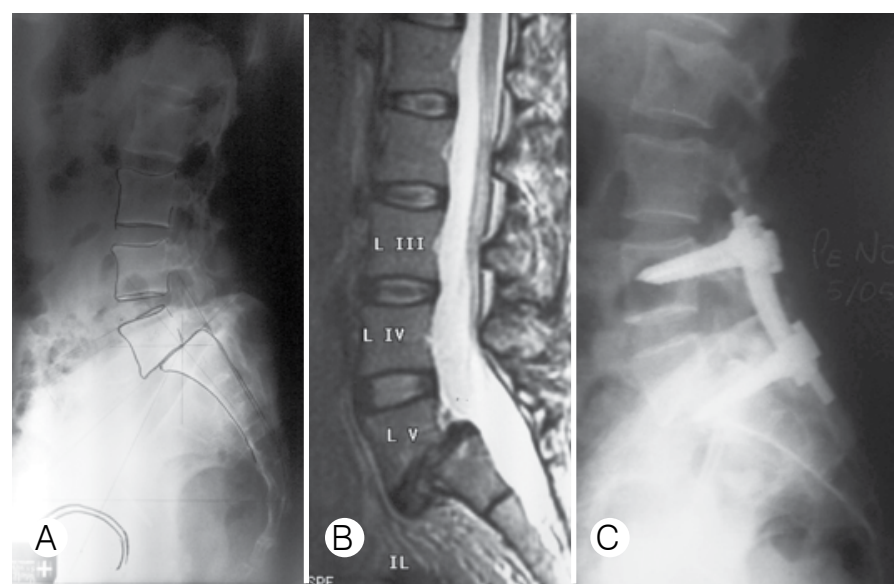

Figura 3. Paciente de 40 años 10 meses, sexo femenino, espondilolistesis grado II, con dolor lumbo-radicular importante (SRS 2). Al seguimiento postoperatorio alejado (7 años 11 meses): sin dolor (SRS 5), vuelta completa a su ocupación previa. (A) Rx perfil Preoperatoria; (B) Resonancia Magnética Preoperatoria; (C) Rx postoperatoria alejada.

\section{CONCLUSIONES}

La descompresión y fusión posterolateral instrumentada in situ permitió más del $80 \%$ de buenos resultados al mediano plazo en la espondilolistesis ístmica lumbosacra del adulto sin importar el grado.

La técnica presentó resultados similares en los parámetros espino-pélvicos del seguimiento a los que se encuentran en los reportes de la literatura con o sin reducción.

\section{REFERENCIAS}

1. Agabegi SS, Fischgrund JS. Contemporary management of isthmic spondylolisthesis: pediatric and adult. Spine J. 2010;10(6):530-43.

2. Jones TR, Rao RD. Adult isthmic spondylolisthesis. J Am Acad Orthop Surg. 2009;17(10):609-17.

3. During J, Goudfrooij H, Keessen W, Beeker TW, Crowe A. Toward standards for posture. Postural characteristics of the lower back system in normal and pathologic conditions. Spine (Phila Pa 1976). 1985;10(1):83-7.

4. Duval-Beaupère G, Schmidt C, Cosson P. A Barycentremetric study of the sagittal shape of spine and pelvis: the conditions required for an economic standing position. Ann Biomed Eng. 1992;20(4):451-62

5. Legaye J, Duval-Beaupère G, Hecquet J, Marty C. Pelvic incidence: a fundamental pelvic parameter for three-dimensional regulation of spinal sagittal curves. Eur Spine J. 1998:7(2):99-103

6. Antoniades SB, Hammerberg KW, DeWald RL. Sagittal plane configuration of the sacrum in spondylolisthesis. Spine (Phila Pa 1976). 2000;25(9):1085-91.

7. Marty C, Boisaubert B, Descamps H, Montigny JP, Hecquet J, Legaye J, et al. The sagittal anatomy of the sacrum among young adults, infants, and spondylolisthesis patients. Eur Spine J. 2002:11(2):119-25.

8. Curylo LJ, Edwards C, DeWald RW. Radiographic markers in spondyloptosis: implications for spondylolisthesis progression. Spine (Phila Pa 1976). 2002;27(18):2021-5.

9. Vaz G, Roussouly P, Berthonnaud E, Dimnet J. Sagittal morphology and equilibrium of pelvis and spine. Eur Spine J. 2002;11(1):80-7.

10. Mac-Thiong JM, Berthonnaud E, Dimar JR 2nd, Betz RR, Labelle H. Sagittal alignment of the spine and pelvis during growth. Spine (Phila Pa 1976). 2004;29(15):1642-7.

11. Labelle H, Roussouly P, Berthonnaud E, Transfeldt E, O'Brien M, Chopin D, et al. SpondyIolisthesis, pelvic incidence, and spinopelvic balance: a correlation study. Spine (Phila Pa 1976). 2004:29(18):2049-54

12. Labelle H, Roussouly P, Berthonnaud E, Dimnet J, O'Brien M. The importance of spinopelvic balance in L5-S1 developmental spondylolisthesis: a review of pertinent radiologic measurements. Spine (Phila Pa 1976). 2005 Mar 15;30(Suppl 6):S27-34.

13. Legaye J, Duval-Beaupère G. Sagittal plane alignment of the spine and gravity: a radiological and clinical evaluation. Acta Orthop Belg. 2005;71(2):213-20.

14. Legaye J. The femoro-sacral posterior angle: an anatomical sagittal pelvic parameter usable with dome-shaped sacrum. Eur Spine J. 2007;16(2):219-25.

15. Vialle $R$, Ilharreborde B, Dauzac C, Lenoir T, Rillardon L, Guigui P. Is there a sagittal imbalance of the spine in isthmic spondylolisthesis? A correlation study. Eur Spine J. 2007:16(10):1641-9

16. Hresko MT, Labelle H, Roussouly P, Berthonnaud E. Classification of high-grade spondylolistheses based on pelvic version and spine balance: possible rationale for reduction. Spine (Phila Pa 1976). 2007;32(20):2208-13.

17. Lafage V, Schwab F, Skalli W, Hawkinson N, Gagey PM, Ondra S, et al. Standing balance and sagittal plane spinal deformity: analysis of spinopelvic and gravity line parameters Spine (Phila Pa 1976). 2008:33(14):1572-8.

18. Schwab F, Lafage V, Patel A, Farcy JP. Sagittal plane considerations and the pelvis in the adult patient. Spine (Phila Pa 1976). 2009;34(17):1828-33.

19. Husson JL, Mallet JF, Parent H, Cavagna R, Vital JM, Blamoutier A, et al. The lumbarpelvic-femoral complex: applications in spinal imbalance. Orthop Traumatol Surg Res. 2010;96(4) Suppl:S1-S9.

20. Le Huec JC, Aunoble S, Philippe L, Nicolas P. Pelvic parameters: origin and significance. Eur Spine J. 2011;20(Suppl 5):564-71.

21. Mac-Thiong JM, Roussouly P, Berthonnaud E, Guigui P. Age- and sex-related variations in sagittal sacropelvic morphology and balance in asymptomatic adults. Eur Spine J. 2011:20(Suppl 5):572-7.

22. Roussouly P, Gollogly S, Berthonnaud E, Labelle H, Weidenbaum M. Sagittal alignment of the spine and pelvis in the presence of L5-s1 isthmic lysis and low-grade spondylolisthesis. Spine (Phila Pa 1976). 2006;31(21):2484-90.

23. Labelle H, Roussouly P, Chopin D, Berthonnaud E, Hresko T, O'Brien M. Spino-pelvic alignment after surgical correction for developmental spondylolisthesis. Eur Spine J. 2008;17(9):1170-6.

24. Kim JS, Lee KY, Lee SH, Lee HY. Which lumbar interbody fusion technique is better in terms of level for the treatment of unstable isthmic spondylolisthesis? J Neurosurg Spine. 2010;12(2):171-7.

25. Park SJ, Lee CS, Chung SS, Kang KC, Shin SK. Postoperative changes in pelvic parameters and sagittal balance in adult isthmic spondylolisthesis. Neurosurgery 2011:68(Suppl 2):355-63

26. Bourghli A, Aunoble S, Reebye O, Le Huec JC. Correlation of clinical outcome and spinopelvic sagittal alignment after surgical treatment of low-grade isthmic spondylolisthesis. Eur Spine J. 2011;20(Suppl 5):663-8.

27. Smith JA, Deviren V, Berven S, Kleinstueck F, Bradford DS. Clinical outcome of transsacral interbody fusion after partial reduction for high-grade L5-S1 spondylolisthesis Spine (Phila Pa 1976). 2001;26(20):2227-34.

28. Boachie-Adjei O, Do T, Rawlins BA. Partial lumbosacral kyphosis reduction, decompression, and posterior lumbosacral transfixation in high-grade isthmic spondylolisthesis 
clinical and radiographic results in six patients. Spine (Phila Pa 1976). 2002;27(6):E161-8.

29. McAfee PC, DeVine JG, Chaput CD, Prybis BG, Fedder IL, Cunningham BW, et al. The indications for interbody fusion cages in the treatment of spondylolisthesis: analysis of 120 cases. Spine (Phila Pa 1976). 2005;30(Suppl 6):S60-5.

30. Floman Y, Millgram MA, Ashkenazi E, Smorgick Y, Rand N. Instrumented slip reduction and fusion for painful unstable isthmic spondylolisthesis in adults. J Spinal Disord Tech. 2008;21(7):477-83.

31. Goyal N, Wimberley DW, Hyatt A, Zeiller S, Vaccaro AR, Hilibrand AS, et al. Radiographic and clinical outcomes after instrumented reduction and transforaminal lumbar interbody fusion of mid and high-grade isthmic spondylolisthesis. J Spinal Disord Tech. 2009;22(5):321-7

32. Giudici F, Minoia L, Archetti M, Corriero AS, Zagra A. Long-term results of the direct repair of spondylolisthesis. Eur Spine J. 2011;20 (Suppl 1):S115-20.

33. Passias PG, Kozanek M, Wood KB. Surgical treatment of low-grade isthmic spondylolisthesis with transsacral fibular strut grafts. Neurosurgery. 2012;70(3):758-63.

34. Ogilvie JW. Complications in spondylolisthesis surgery. Spine (Phila Pa 1976). 2005;30(Suppl 6):S97-101.

35. Sansur CA, Reames DL, Smith JS, Hamilton DK, Berven SH, Broadstone PA, et al. Morbidity and mortality in the surgical treatment of 10,242 adults with spondylolisthesis. $J$ Neurosurg Spine. 2010;13(5):589-93.

36. Lenke LG, Bridwell KH, Bullis D, Betz RR, Baldus C, Schoenecker PL. Results of in situ fusion for isthmic spondylolisthesis. J Spinal Disord. 1992;5(4):433-42.

37. Butt MF, Dhar SA, Hakeem I, Faroog M, Halwai MA, Mir MR, et al. In situ instrumented posterolateral fusion without decompression in symptomatic low-grade isthmic spondylolisthesis in adults. Int Orthop. 2008;32(5):663-9.

38. Jacobs WC, Vreeling A, De Kleuver M. Fusion for low-grade adult isthmic spondylolisthesis: a systematic review of the literature. Eur Spine J. 2006;15(4):391-402.

39. Carragee EJ. Single-level posterolateral arthrodesis, with or without posterior decompression, for the treatment of isthmic spondylolisthesis in adults. A prospective, randomized study. J Bone Joint Surg Am. 1997;79(8):1175-80.

40. Schnee CL, Freese A, Ansell LV. Outcome analysis for adults with spondylolisthesis treated with posterolateral fusion and transpedicular screw fixation. J Neurosurg. 1997;86(1):56-63.

41. Deguchi M, Rapoff AJ, Zdeblick TA. Posterolateral fusion for isthmic spondylolisthesis in adults: analysis of fusion rate and clinical results. J Spinal Disord. 1998;11(6):459-64.
42. Wenger M, Sapio N, Markwalder TM. Long-term outcome in 132 consecutive patients after posterior internal fixation and fusion for Grade I and II isthmic spondylolisthesis. J Neurosurg Spine. 2005;2(3):289-97.

43. Suda K, Ito M, Abumi K, Haba H, Taneichi H, Kaneda K. Radiological risk factors of pseudoarthrosis and/or instrument breakage after PLF with the pedicle screw system in isthmic spondylolisthesis. J Spinal Disord Tech. 2006;19(8):541-6.

44. Dahl B, Gehrchen P, Blyme P, Kiaer T, Tøndevold E. Clinical outcome after spinal fusion with a rigid versus a semi-rigid pedicle screw system. Eur Spine J. 1997;6(6):412-6.

45. Swan J, Hurwitz E, Malek F, van den Haak E, Cheng I, Alamin T, et al. Surgical treatment for unstable low-grade isthmic spondylolisthesis in adults: a prospective controlled study of posterior instrumented fusion compared with combined anterior-posterior fusion. Spine J. 2006;6(6):606-14.

46. Ekman $P$, Möller $H$, Tullberg T, Neumann $P$, Hedlund R. Posterior lumbar interbody fusion versus posterolateral fusion in adult isthmic spondylolisthesis. Spine (Phila Pa 1976). 2007;32(20):2178-83

47. Müslüman AM, Yılmaz A, Cansever T, Cavu o lu H, Colak I, Genç HA, Aydın Y. Posterior lumbar interbody fusion versus posterolateral fusion with instrumentation in the treatment of low-grade isthmic spondylolisthesis: midterm clinical outcomes. J Neurosurg Spine. 2011;14(4):488-96.

48. Benli IT, Ciçek H, Kaya A. Comparison of sagittal plane realignment and reduction with posterior instrumentation in developmental low or high dysplastic spondylolisthesis. Kobe J Med Sci. 2006;52(6):151-69.

49. Kim JS, Kim DH, Lee SH, Park CK, Hwang JH, Cheh G, et al. Comparison study of the instrumented circumferential fusion with instrumented anterior lumbar interbody fusion as a surgical procedure for adult low-grade isthmic spondylolisthesis. World Neurosurg. 2010;73(5):565-71.

50. Hresko MT, Hirschfeld R, Buerk AA, Zurakowski D. The effect of reduction and instrumentation of spondylolisthesis on spinopelvic sagittal alignment. J Pediatr Orthop. 2009;29(2):157-62

51. Hanson DS, Bridwell KH, Rhee JM, Lenke LG. Correlation of pelvic incidence with lowand high-grade isthmic spondylolisthesis. Spine (Phila Pa 1976). 2002;27(18):2026-9.

52. Boulay C, Tardieu C, Hecquet J, Benaim C, Mouilleseaux B, Marty C, et al. Sagittal alignment of spine and pelvis regulated by pelvic incidence: standard values and prediction of lordosis. Eur Spine J. 2006;15(4):415-22. 